\title{
Changes In vertical dimension: Extraction versus non-extraction
}

\author{
Dr Khulood Alhajeri,' Dr Pratik Premjani,2 Dr Ahmed Ismail,3 Dr Donald Ferguson4 \\ iSpecialist Orthodontist, 2Faculty, Department of Orthodontics, European University, College \\ 3Professor, Department of Orthodontics, European University, College 4Professor/Dean, European University, College,
}

Dubai, UAE,

Correspondence: Dr Donald J. Ferugson; Email: donald.ferguson@euc.ac.ae

\section{ABSTRACT}

Introduction: To evaluate and compare the vertical changes in patients treated orthodontically with or without different patterns of premolars extraction.

Materials \& Method: The lateral cephalograms of 112 orthodontically treated patients were categorized into four groups of 28 each based upon extraction strategy. Cephalometric analysis comprised of 3 angular and 8 linear measurements were used to analyze vertical changes from pre- to post treatment.

Result: Active orthodontic treatment with or without various premolar extraction therapies led to a statistically significantly increase in the anterior facial height. Second premolar extraction resulted in significantly greater forward movement of the maxillary and mandibular first molars compared to non-extraction therapy.

Conclusion: Active orthodontic treatment resulted in no significant changed in maxillary to mandibular (MM) angle with or without extraction therapy.

Keywords: Extraction, First premolar, Non-extraction, Second premolar, Vertical dimension.

\section{INTRODUCTION}

The extraction of premolars as a practical form of orthodontic therapy has been accepted for many years, the indications for first premolars extraction are usually severe anterior crowding or lip protrusion, while in borderline cases with moderate crowding, fairly wellaligned incisors, and a relatively acceptable profile, second premolars can be extracted,' or to close down the bite like Schudy ${ }^{2-4}$ described facial types as "hypo- and hyper divergence" and recommended a non-extraction approach in the treatment of hypo divergent facial types and an extraction approach "to close down the bite" in hyper divergent types.

Sassouni and $\mathrm{Nanda}^{5}$ concurred with this treatment philosophy. If molars move forward without extrusion to the extraction spaces, by the principle of "wedge effect will forward rotate the mandible resulting in vertical dimension decrease. ${ }^{2-6}$ (Figure 1) However, there is great controversy concerning the effects of premolar extractions on facial vertical dimension (FVD).

Chua et $\mathrm{al}^{7}$ found that premolar extraction was not associated with any significant change of the lower anterior facial height (LAFH), whereas non-extraction treatment was associated with a significant increase in $\mathrm{LAFH}$.
Staggers ${ }^{6}$ and Kocadereli $^{8}$ found that the vertical changes that occurred after the extraction of first premolars were not different from those that occurred in the non-extraction cases. However, in these two studies, it was pointed out that there was minimal need for protraction of posterior teeth because most of the extraction space was used to relieve crowding or to retract the anterior teeth. It was suggested that the absence of posterior teeth protraction could explain the comparable changes in the vertical dimension between extraction and non-extraction groups.

Al-Nimri9 found that Mandibular premolar extraction in Class II division 1 subjects was not associated with a significant reduction of the facial divergence measured by the MM angle and the MP angle. However the Second premolar extraction was associated with more forward movement of the mandibular molars.

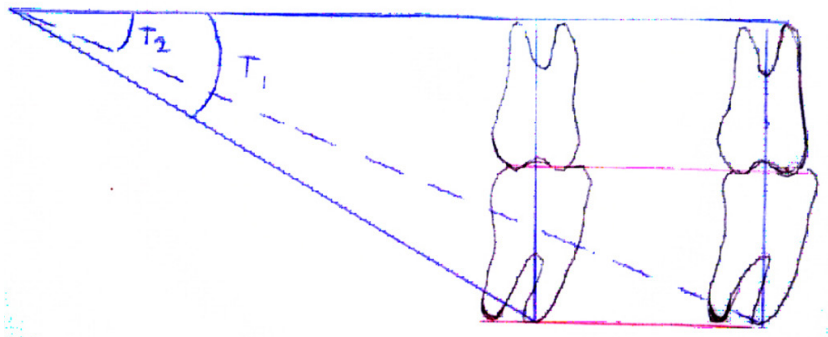

Figure 1: Hypothesis of moving the molar forward. 
Sharma ${ }^{10}$ study indicates that the orthodontic treatment of class II division 1 malocclusion cases treated either with a non-extraction approach or with the extraction of all first premolars, lead to a statistically significantly increase in the anterior facial height.

The purpose of this study was to evaluate and compare the vertical changes occurring in patients treated orthodontically without or with different patterns of premolars extraction.

\section{MATERIALS AND METHOD}

In this retrospective study, the sample was selected from the archived patient care records of 112 patients aged of 11 to 49 years, each group consist of 13 adult and 15 growing. They considered as adult if the cervical vertebral of the patient in cervical stage $6^{11}$ (CS6: The concavities at the lower borders of $C 2, C 3$, and C4 still are evident. At least one of thebodies of C3 and C4 is rectangular vertical in shape). All the patients treated in the Orthodontics Department of European University College with pre-adjusted MBT, slot size 0.022 inch.

The subjects were selected on the basis of the following criteria:

- The availability of pre and post treatment lateral cephalometric radiographs.

- No headgear or functional appliance was used before or during the fixed appliance therapy.

- Extraction space completely closed in post treatment lateral cephalometric radiographs.

The patients were grouped into four equal groups of 28 subjects each:

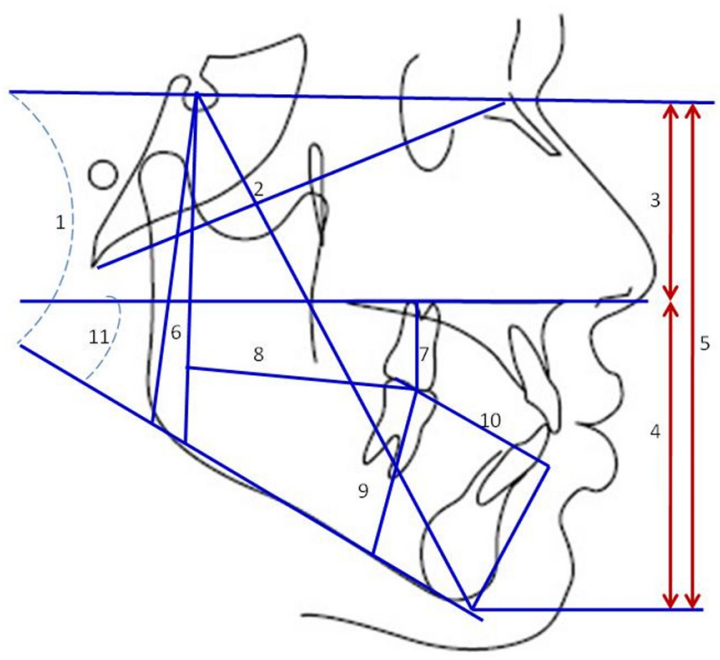

Group 1: Non-extraction sample was comprised of 13 males and 15 females with pretreatment age from 12 to 45 years.

Group 2: Extraction sample of upper first premolar and lower second premolar (U4/L5), was comprised of 6 males and 22 females with pretreatment age from 12 to 45 years.

Group 3: Extraction sample of upper first premolar and lower first premolars (U4/L4), was comprised of 13 males and 22 females with pretreatment age from 12 to 45 years.

Group 4: Extraction sample of upper second premolars and lower second premolars (U5/L5), was comprised 11 Male and 22 female) with pretreatment age from 11 to 49 years

All cephalometric radiographs were taken on the same cephalostat. The cephalometric analysis included a manual tracing for 12 measurements selected to evaluate vertical changes: 3 angular, 8 linear and 1 ratio. (Figure 2)

Cephalometric radiographs pre and post treatment of twenty two patients were randomly selected and traced after 2 weeks, which show no significant different (Reliability Testing)

For Descriptive statistics, the mean values with standard deviations were calculated Table I. Measurements comparing pretreatment cephalometric radiographs and post-treatment cephalometric radiographs were tested statistically using paired t-tests (table II,III,IV,V.) In all tests, the significance level was $P<0.05$. ANOVA Test was used to compare between the 4 groups table $\mathrm{VI}$ and to compare between 8 groups table VII.

$1=$ SN-GoGn angle $; 2=$ SGn-NBa angle $; 3=$ Na-ANS $\mathrm{mm}$ (upper face height); 4 = ANS-Me $\mathrm{mm}$ (lower face height); 5 = Na-Me $\mathrm{mm}$ (anterior face height); 6 = S-Go $\mathrm{mm}$ (posterior face height); 7 = UM to palatal plane $\mathrm{mm}$ (vertical distance from bisect of maxillary first molar occlusal surface to palatal plane); 8 = UM to occlusal surface of maxillary first molar to $\mathrm{SN}$ perpendicular; $9=$ LM to mandibular plane (vertical distance from bisect of mandibular first molar occlusal surface to mandibular plane; $10=$ LM to Gn horizontal (horizontal distance from bisect of mandibular first molar occlusal surface to perpendicular to mandibular at gnathion); 11 = PP-GoGn angle (palatal plane to mandibular plane angle).

Figure $2^{12}$ : Cephalometric analysis used in the study of vertical dento-facial changes. 


\section{RESULT}

Descriptive statistics were computed for age and each cephalometric variable at pre-treatment ( $\mathrm{T} 1$ ) and post treatment (T2) for each of the four study groups by adult and non adult subjects. (Table 1)

Paired t-test was used for within group changes to determine differences due to active treatment ( $\mathrm{T} 1$ to T2) for all four study groups (Tables 2, 3, 4 and 5). In the non extraction Group 1 there were a significantly increase in the lower facial height (ANS-Me) from pretreatment (69.63 \pm 4.69$)$ to post treatment (71.50 \pm 5.80$)$, significant increase in totally facial height (NA-Me) from pre-treatment (127.39 \pm 6.83 ) to post treatment (129.70 \pm 7.17 ), and posterior facial height (S-Go) significantly increased from pre-treatment (83.71 \pm 5.80$)$ to post treatment (85.96 \pm 6.09 ). Upper first molar also significant extruded (Um-Palatal plane) from pre $(26.91 \pm 2.86)$ to post treatment $27.95+/-2.26$ ) and moved significantly forward (Um-SN horizontal) from pre (34.57 \pm 5.69$)$ to post treatment (36.66 \pm 5.88 ). (Table 2 and Figure 3 )

In U4/L5 extraction Group 2, there was a significant decrease in SGn-Nba from pre $(89.7 \pm 7.21)$ to post treatment $(89.1 \pm 6.95)$ which meant the mandible rotated downward and backward. There were significant increases in lower facial height (ANS-Me) from pre (66.50 \pm 5.37$)$ to post treatment (68.6 \pm 5.78$)$, total anterior facial height ( $\mathrm{Na}-\mathrm{Me}$ ) from pre (121.50 $\pm 5.76)$ to post treatment $(124.10 \pm 6.11)$ and posterior facial height (S-Go) significantly increased from pre

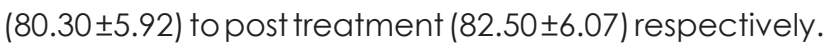
Upper first molar significantly extruded (Um-Palatal plane) from pre $(25.50 \pm 2.05)$ to post treatment $(29.94$ \pm 1.95 ) and also moved significantly forward (Um-SN

Table 1: Descriptive statistics including mean, standard deviation (SD) for age and each cephalometric variables at pre-treatment (T1) and post treatment ()T2) for each of the four study groups by adult and non adult subjects.

\begin{tabular}{|c|c|c|c|c|c|c|c|c|c|c|c|c|c|c|c|c|}
\hline \multirow{3}{*}{ Variable } & \multicolumn{4}{|c|}{ Group 1:Non Extraction } & \multicolumn{4}{|c|}{ Group 2: Extraction U4/L5 } & \multicolumn{4}{|c|}{ Group 3: Extraction U4/U4 } & \multicolumn{4}{|c|}{ Group 4: Extraction U5/L5 } \\
\hline & \multicolumn{2}{|c|}{ Adult } & \multicolumn{2}{|c|}{ Non-Adult } & \multicolumn{2}{|c|}{ Adult } & \multicolumn{2}{|c|}{ Non-Adult } & \multicolumn{2}{|c|}{ Adult } & \multicolumn{2}{|c|}{ Non-Adult } & \multicolumn{2}{|c|}{ Adult } & \multicolumn{2}{|c|}{ Non-Adult } \\
\hline & mean & SD & mean & SD & mean & SD & mean & SD & mean & SD & mean & SD & mean & SD & mean & SD \\
\hline AgeTl & & & & & & & & & & & & & & & & \\
\hline Aget2 & & & & & & & & & & & & 1.36 & & 10.98 & 16.04 & 1.78 \\
\hline SN-GoGn angleTI & 32.42 & 4.28 & & & & & & & & & & & & & & 4.69 \\
\hline SN-GoGn angleT2 & & 4.40 & & & & & & 7.27 & & & & 6.00 & & & & 4.93 \\
\hline SGn-Nba angleT1 & & 13.54 & & & & & & 3.93 & & & & & & & & 7.20 \\
\hline SGn-Nba a & & & & & & & & & & & & & & & & 10.46 \\
\hline Na-ANS $(m m) T 1$ & 57.77 & 3.09 & & 5.68 & & & & 3.68 & & & & 2.35 & & & & 5.33 \\
\hline Na-ANS $(\mathrm{mm}) \mathrm{T} 2$ & & & & & & & & & & & & & & & & 4.73 \\
\hline ANS-Me $(\mathrm{mm}) \mathrm{Tl}$ & & 4.50 & 67.73 & & 67.23 & & 65.80 & 4.89 & & 5.13 & 66.53 & 6.01 & 72.04 & 7.32 & 68.67 & 7.31 \\
\hline ANS-Me $(\mathrm{mm}) \mathrm{T} 2$ & 73.23 & 5.15 & 70.00 & 6.09 & 68.42 & 5.71 & 68.70 & 6.04 & 70.27 & 4.68 & 68.80 & 6.12 & 72.04 & 7.62 & 71.40 & 8.15 \\
\hline $\mathrm{Na}-\mathrm{Me}(\mathrm{mm}) \mathrm{Tl}$ & 129.58 & 4.83 & & 7.86 & 22.50 & 5.75 & 105.60 & 38.16 & 126.27 & 5.01 & 124.03 & 6.86 & 127.42 & 9.32 & 126.80 & 11.42 \\
\hline & & 5.19 & & 8.58 & & 5.69 & & 39.71 & & 4.71 & & 7.37 & & & & 11.63 \\
\hline & & 6.79 & & 3.31 & & & & 7.00 & & & & & & & & 6.65 \\
\hline $\mathrm{S}-\mathrm{Go}(\mathrm{mm}) \mathrm{T} 2$ & & 6.52 & & 5.51 & & & 82.30 & 7.69 & & 4.05 & 84.77 & 8.14 & & 6.02 & 86.67 & 7.28 \\
\hline ANS-Me/Na-Me (\%)TI & 55.40 & 2.27 & 54.08 & 2.65 & 54.83 & 3.70 & 54.53 & 2.58 & 55.56 & 3.11 & 52.87 & 3.54 & 56.45 & 2.25 & 54.27 & 5.16 \\
\hline ANS-Me/Na-Me (\%)T2 & 55.94 & 2.46 & 53.37 & 2.65 & 55.29 & 3.53 & 55.21 & 3.08 & 55.52 & 2.92 & 53.30 & 3.29 & 56.44 & 2.39 & 55.20 & 5.53 \\
\hline UM-PalatalPlane $(\mathrm{mm}) \mathrm{Tl}$ & 28.15 & 1.68 & 25.83 & 3.27 & 26.23 & 1.86 & 24.87 & 2.07 & 27.92 & 2.73 & 25.73 & 3.01 & 27.89 & 2.82 & 25.40 & 2.50 \\
\hline UM-PalatalPlane (mm)T2 & 28.81 & & & 2.29 & & & & & & & & 5.28 & & & & 2.75 \\
\hline UM-SN horizontal $(\mathrm{mm}) \mathrm{Tl}$ & 33.47 & 11.36 & 33.40 & 4.23 & 37.08 & 5.22 & 34.40 & 6.61 & 34.65 & 4.22 & 35.87 & 6.59 & 33.27 & 6.70 & 35.37 & 7.45 \\
\hline UM-SN horizontal $(\mathrm{mm}) \mathrm{T} 2$ & 37.12 & 7.01 & 36.27 & 4.95 & & & 39.30 & 6.51 & & 4.38 & 41.30 & 6.79 & 37.42 & 6.60 & 41.40 & 8.00 \\
\hline LM-MandPlane $(\mathrm{mm}) \mathrm{T} 1$ & 29.05 & 8.22 & 29.83 & 2.16 & 31.50 & 2.69 & & 3.12 & & 2.78 & & 2.50 & & 3.75 & & 3.79 \\
\hline LM-MandPlane $(\mathrm{mm}) \mathrm{T} 2$ & 32.19 & 2.40 & 30.50 & 2.69 & 32.85 & 3.06 & 32.07 & 2.70 & 32.92 & 2.27 & 32.23 & 2.69 & 33.12 & 3.73 & 33.00 & 4.31 \\
\hline LM-GnHorizontalT1 & 38.21 & 11.36 & 37.37 & 1.37 & 35.12 & 4.01 & 36.03 & 3.74 & 34.77 & 3.11 & 35.30 & 2.85 & 36.69 & 3.00 & 37.67 & 3.66 \\
\hline LM-GnHorizontalT2 & 40.71 & 5.09 & 37.67 & 2.26 & 32.27 & 4.64 & 32.63 & 3.58 & 32.77 & 3.73 & 32.83 & 3.90 & 33.23 & 3.24 & 32.57 & 4.55 \\
\hline PP-Go Gn angle & 25.77 & 3.66 & 25.93 & 7.38 & 25.62 & & 28.93 & 5.61 & 30.92 & 6.49 & 24.47 & 4.73 & 30.12 & 5.17 & 28.37 & 5.29 \\
\hline PP-Go Gn angle & 26.04 & 3.62 & 25.67 & 7.37 & 26.12 & 6.59 & 30.43 & 8.18 & 30.77 & 6.37 & 24.33 & 4.24 & 29.96 & 5.23 & 28.43 & 5.64 \\
\hline
\end{tabular}


Table 2: Results of pair t-testing for Group 1 (non-extraction) showing treatment effects, i.e. from pre treatment to post treatment. Note that 5 of 12 measurements were significantly different.

\begin{tabular}{|c|c|c|c|c|c|c|}
\hline \multirow{2}{*}{$\begin{array}{l}\text { Group 1: Non Extraction } \\
\text { Cephalomatric variables }\end{array}$} & \multicolumn{2}{|c|}{ Pre-treatment } & \multicolumn{2}{|c|}{ Post-treatment } & \multirow{2}{*}{ mean dif } & \multirow{2}{*}{ p signifi } \\
\hline & mean & SD & mean & SD & & \\
\hline SN-GoGn angle & 32.30 & 5.00 & 32.07 & 4.59 & -0.23 & NS \\
\hline SGn-Nba angle & 92.03 & 4.22 & 92.28 & 4.15 & 0.25 & NS \\
\hline Na-ANS(mm) & 57.76 & 4.58 & 58.21 & 3.87 & 0.44 & NS \\
\hline ANS-Me(mm) & 69.62 & 4.69 & 71.50 & 5.80 & 1.87 & $P<0.001$ \\
\hline $\mathrm{Na}-\mathrm{Me}(\mathrm{mm})$ & 127.39 & 6.83 & 129.69 & 7.17 & 2.30 & $P<0.001$ \\
\hline S-Go(mm) & 83.71 & 5.80 & 85.96 & 6.09 & 2.25 & $P<0.001$ \\
\hline ANS-Me/Na-Me (\%) & 54.69 & 2.52 & 54.56 & 2.83 & -0.13 & NS \\
\hline UM-palatal plane (mm) & 26.91 & 2.86 & 27.94 & 2.26 & 1.03 & $P<0.05$ \\
\hline UM-SN horizontal(mm) & 34.57 & 5.69 & 36.66 & 5.88 & 2.08 & $P<0.001$ \\
\hline LM-mandibular plane & 30.48 & 2.45 & 31.28 & 2.65 & 0.80 & NS \\
\hline LM-Gn horizontal & 39.10 & 3.94 & 39.07 & 4.06 & -0.02 & NS \\
\hline PP-Go Gn angle & 25.85 & 5.85 & 25.83 & 5.83 & -0.017 & NS \\
\hline
\end{tabular}

Table 3: Results of pair t-testing for Group 1 (non-extraction) showing treatment effects, i.e. from pre treatment to post treatment. Note that 5 of 12 measurements were significantly different.

\begin{tabular}{|c|c|c|c|c|c|c|}
\hline \multirow{2}{*}{$\begin{array}{c}\text { Group 2: Ext U4/L5 } \\
\text { Cephalomatric variables }\end{array}$} & \multicolumn{2}{|c|}{ Pre-treatment } & \multicolumn{2}{|c|}{ Post-treatment } & \multirow{2}{*}{ mean dif } & \multirow{2}{*}{ p signifi } \\
\hline & mean & SD & mean & SD & & \\
\hline SN-GoGn angle & 33.82 & 6.1 & 34.01 & 6.26 & 0.19 & NS \\
\hline SGn-Nba angle & 89.69 & 7.21 & 89.12 & 6.95 & -0.57 & $P<0.01$ \\
\hline Na-ANS (mm) & 54.67 & 3.54 & 55.17 & 3.88 & 0.50 & $P<0.01$ \\
\hline ANS-Me(mm) & 66.46 & 5.37 & 68.57 & 5.78 & 2.10 & $P<0.001$ \\
\hline $\mathrm{Na}-\mathrm{Me}(\mathrm{mm})$ & 121.48 & 5.76 & 124.05 & 6.11 & 2.57 & $P<0.001$ \\
\hline S-Go(mm) & 80.30 & 5.92 & 82.51 & 6.07 & 2.21 & $P<0.01$ \\
\hline ANS-Me/Na-Me (\%) & 54.66 & 3.09 & 55.24 & 3.23 & -0.57 & $P<0.01$ \\
\hline UM-palatal plane(mm) & 25.50 & 2.05 & 26.94 & 1.95 & 1.44 & $P<0.001$ \\
\hline UM-SN horizontal (mm) & 35.64 & 6.05 & 39.67 & 5.75 & 4.03 & $P<0.001$ \\
\hline LM-mandibular plane & 30.30 & 3.09 & 32.42 & 2.84 & 2.12 & $P<0.001$ \\
\hline LM-Gn horizontal & 35.60 & 3.82 & 32.46 & 4.03 & -3.14 & $P<0.001$ \\
\hline PP-Go Gn angle & 27.39 & 6.15 & 27.71 & 6.11 & 0.32 & NS \\
\hline
\end{tabular}

horizontal) from pre $(35.60 \pm 6.05)$ to post treatment (39.70 \pm 5.75$)$. Lower first molar significantly extruded (Lm-Mandibular plane) from pre $(30.30 \pm 3.09)$ to post treatment $(32.40 \pm 2.84)$ and also moved significantly forward ( $\mathrm{Lm}-\mathrm{Gn}$ horizontal) form pre $(35.60 \pm 6.15)$ to post treatment ( $32.50 \pm 6.11$ ). (Table 3 and Figure 4)

In Group 3 U4/L4 extraction, there were significant increases in the lower facial height (ANS-Me) from pre $(68.21 \pm 5.81)$ to post treatment $(69.48 \pm 5.44)$, total anterior facial height (Na-Me) from pre (125.07 \pm 6.06 ) to post treatment (126.61 \pm 6.16$)$ and posterior facial height (S-Go) significantl increased from pre $(82.02$ \pm 5.41 ) to post treatment (84.29 \pm 6.47$)$. Upper first molar significantly extruded (Um-Palatal plane) from pre $(26.75 \pm 3.03)$ to post treatment $(28.21 \pm 4.17)$ and also moved significantly forward (Um-SN horizontal) from pre $(35.30 \pm 5.55)$ to post treatment $(39.70 \pm 5.96)$. Lower first molar significantly extruded (Lm-Mandibular plane) from pre $(30.71 \pm 2.83)$ to post treatment (32.55 \pm 2.48 ) and also moved significantly forward (Lm-Gn 


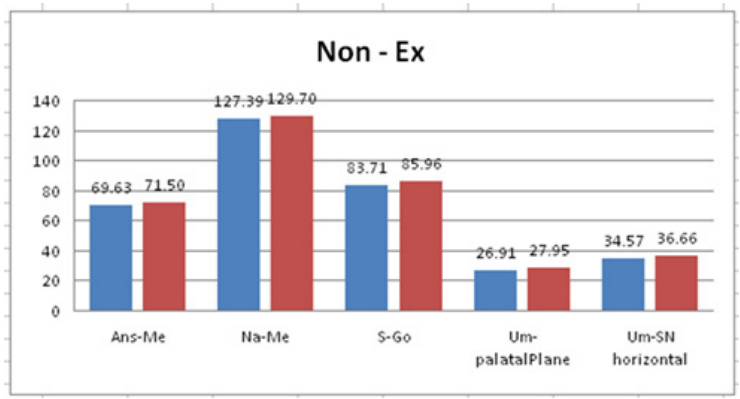

Figure 3: Bar chart for Group 1 (non-extraction) demonstrating which cephalometric variables were significantly different between pre- and post treatment.

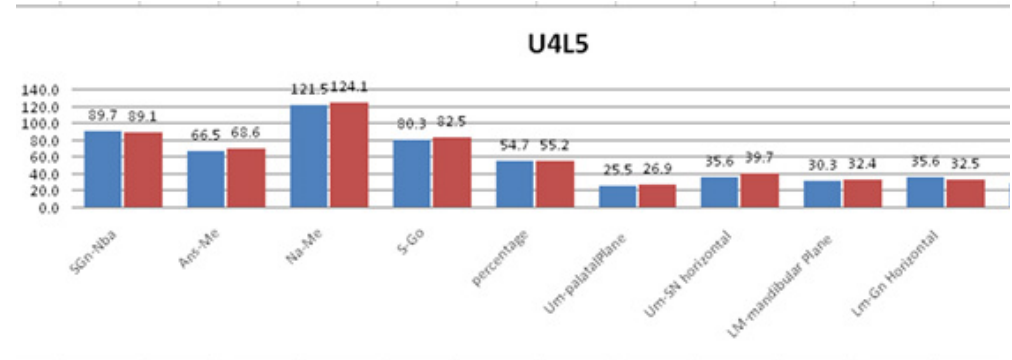

Figure 4: Bar chart for Group 1 (ext U4/L5) demonstrating which cephalometric variables were significantly different between pre- and post treatment.

Table 4: Results of pair t-testing for Group 1 (ext U4/L4) showing treatment effects, i.e. from pre treatment to post treatment. Note that 7 of 12 measurements were significantly different.

\begin{tabular}{|c|c|c|c|c|c|c|}
\hline \multirow{2}{*}{$\begin{array}{c}\text { Group 3: Ext U4/L4 } \\
\text { Cephalomatric variables }\end{array}$} & \multicolumn{2}{|c|}{ Pre-treatment } & \multicolumn{2}{|c|}{ Post-treatment } & \multirow{2}{*}{ mean dif } & \multirow{2}{*}{ p signifi } \\
\hline & mean & SD & mean & SD & & \\
\hline SN-GoGn angle & 34.51 & 5.38 & 33.94 & 5.80 & -0.57 & NS \\
\hline SGn-Nba angle & 90.33 & 5.88 & 90.26 & 6.14 & -0.07 & NS \\
\hline Na-ANS(mm) & 56.92 & 3.22 & 57.26 & 3.44 & 0.33 & NS \\
\hline ANS-Me(mm) & 68.21 & 5.81 & 69.48 & 5.44 & 1.26 & $P<0.01$ \\
\hline $\mathrm{Na}-\mathrm{Me}(\mathrm{mm})$ & 125.07 & 6.06 & 126.60 & 6.16 & 1.53 & $P<0.01$ \\
\hline S-Go(mm) & 82.01 & 5.41 & 84.28 & 6.47 & 2.26 & $P<0.001$ \\
\hline ANS-Me/Na-Me (\%) & 54.11 & 3.56 & 54.33 & 3.26 & 0.21 & NS \\
\hline UM-palatal plane(mm) & 26.75 & 3.03 & 28.21 & 4.17 & 1.46 & $P<0.01$ \\
\hline UM-SN horizontal(mm) & 35.30 & 5.55 & 39.69 & 5.96 & 4.39 & $P<0.001$ \\
\hline LM-mandibular plane & 30.71 & 2.83 & 32.55 & 2.48 & 1.83 & $P<0.001$ \\
\hline LM-Gn horizontal & 35.05 & 2.9 & 32.80 & 3.75 & -2.25 & $P<0.001$ \\
\hline PP-Go Gn angle & 27.46 & 6.40 & 27.32 & 6.61 & -0.14 & NS \\
\hline
\end{tabular}

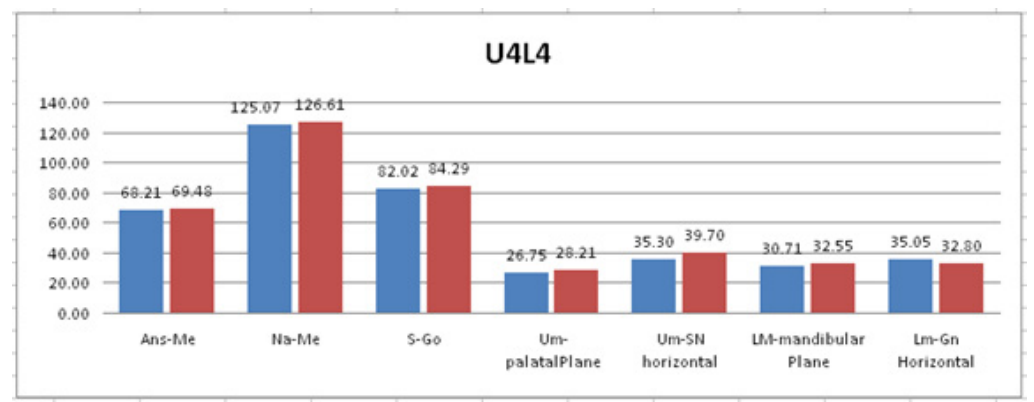

Figure 5: Bar chart for Group 3 (ext U4/L4) demonstrating which cephalometric variables were significantly different between pre- and post treatment. 
Table 5: Results of pair t-testing for Group 1 (ext U5/L5) showing treatment effects, i.e. from pre treatment to post treatment. Note that 9 of 12 measurements were significantly different.

\begin{tabular}{|c|c|c|c|c|c|c|}
\hline \multirow{2}{*}{$\begin{array}{c}\text { Group 4: Ext U5/L5 } \\
\text { Cephalomatric variables }\end{array}$} & \multicolumn{2}{|c|}{ Pre-treatment } & \multicolumn{2}{|c|}{ Post-treatment } & \multirow{2}{*}{ mean dif } & \multirow{2}{*}{ p signifi } \\
\hline & mean & SD & mean & SD & & \\
\hline SN-GoGn angle & 34.76 & 4.59 & 34.32 & 4.78 & -0.44 & $\mathrm{P}<0.05$ \\
\hline SGn-Nba angle & 91.12 & 4.56 & 91.46 & 4.42 & 0.33 & Ns \\
\hline Na-ANS (mm) & 56.85 & 4.66 & 57.01 & 4.36 & 0.16 & Ns \\
\hline ANS-Me(mm) & 70.23 & 7.37 & 71.69 & 7.76 & 1.46 & $P<0.001$ \\
\hline $\mathrm{Na}-\mathrm{Me}(\mathrm{mm})$ & 127.08 & 10.31 & 128.71 & 10.58 & 1.62 & $P<0.001$ \\
\hline S-Go(mm) & 83.08 & 6.23 & 85.03 & 6.83 & 1.94 & $P=0.001$ \\
\hline ANS-Me/Na-Me (\%) & 55.28 & 4.15 & 55.77 & 4.33 & 0.49 & $P<0.01$ \\
\hline UM-palatal plane (mm) & 26.55 & 2.89 & 27.76 & 2.73 & 1.21 & $P<0.001$ \\
\hline UM-SN horizontal (mm) & 34.39 & 7.06 & 39.55 & 7.52 & 5.16 & $P<0.001$ \\
\hline LM-mandibular plane & 31.23 & 3.78 & 33.05 & 23.97 & 1.82 & $P<0.001$ \\
\hline LM-Gn horizontal & 37.21 & 3.34 & 32.87 & 3.94 & -4.33 & $P<0.001$ \\
\hline PP-Go Gn angle & 29.17 & 5.21 & 29.14 & 5.40 & -0.035 & Ns \\
\hline
\end{tabular}

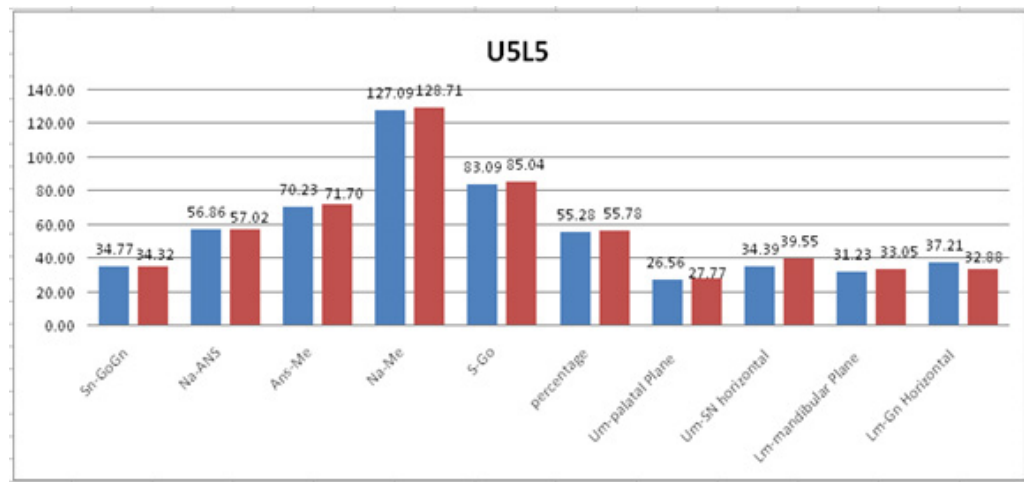

Figure 6: Bar chart for Group 3 (ext U5/L5) demonstrating which cephalometric variables were significantly different between pre- and post treatment.

horizontal) from pre (35.05 \pm 2.9$)$ to post treatment (32.80 \pm 3.75 ). The angular measurements (SGn-Nba, SN-GoGN, PP-Gn) did not significantly change. (Table 4 and Figure 5)

In extraction U5/L5 Group 4, there was a significant decrease in SN-GoGN from pre $(34.77 \pm 4.59$, to post treatment $(34.32 \pm 4.78)$, which meant anterior rotation of the mandible. There were significant increases in the lower facial height (ANS-Me) from pre $(70.23 \pm 7.37)$ to post treatment $(71.70 \pm 7.76)$, total anterior facial height (Na-Me) significantly increased from pre (127.09 \pm 10.31 ) to post treatment $(128.71 \pm 10.58)$ and posterior facial height ( $\mathrm{S}-\mathrm{GO}$ ) significantly increased from pre (83.09 \pm 6.23 ) to post treatment (85.04 \pm 6.83 ). Upper first molar significantly extruded (Um-Palatal plane) from pre $(26.56 \pm 2.89)$ to post treatment $(27.77 \pm 2.73)$ and also moved significantly forward (Um-SN horizontal) from pre (34.39 \pm 7.06$)$ to post treatment (39.55 \pm 7.52$)$. Lower first molar significantly extruded (Lm-Mandibular plane) from pre $(31.23 \pm 3.78)$ to post treatment (33.05 \pm 3.97 ) and also significantly moved forward (Lm-Gn horizontal) from pre $(37.21 \pm 3.34)$ to post treatment (32.88 \pm 3.94 ). The angular measurements (SGn-Nba, PP-Gn) did not significantly change. (Table 5 and Figure 6). 
Table 6: Results of ANOVA testing showed that mesial movement of the first molars for group 5 (U5/L5) moved significantly more forward than group 1, non extraction (Non Ext). ${ }^{*}=\mathrm{P}=.001$.

\begin{tabular}{|l|c|c|c|c|c|c|c|c|c|}
\hline \multirow{2}{*}{$\begin{array}{c}\text { Group 4: Ext U5/L5 } \\
\text { Cephalomatric variables }\end{array}$} & \multicolumn{2}{|c|}{ Group 1 (Non Ext) } & \multicolumn{2}{c|}{ Group 2 (U4/L5) } & \multicolumn{2}{c|}{ Group 3 (U4/L4) } & \multicolumn{2}{c|}{ Group 4 (U5/L5) } \\
\cline { 2 - 11 } & mean & SD & mean & SD & mean & SD & mean & SD & p signifi \\
\hline UM-SN horizontal & $2.08^{*}$ & 1.70 & 4.03 & 2.60 & 4.30 & 3.35 & $5.16^{*}$ & 2.03 & P=.001 \\
\hline LM-Gn horizontal & $-.02^{*}$ & 2.00 & -3.14 & 1.76 & -2.25 & 2.53 & $-4.33^{*}$ & 3.13 & $P=.001$ \\
\hline
\end{tabular}
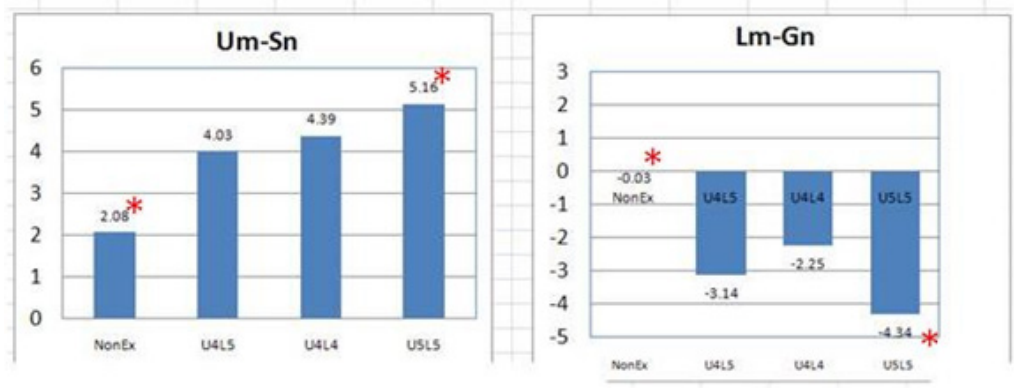

Figure 7: Bar chart demonstrating significantly more mesial movement of upper first molar (Um-Sn) and lower first molar (Lm-Gn) at post treatment when extraction of all second premolars (U5/L5) was used compared to non-extraction (NonExt). * $=\mathrm{P}=.001$

One way ANOVA testing was used to determine significant differences in post treatment position of first molars among the four groups. Significant differences were found between Groups 1 and 4 in first molar position at post treatment. The upper first molars moved significantly more forward (Um-Sn) in U5/L5 Group 4 (5.16 \pm 2.03$)$ compared to non-extraction Group 1 (2.08 $\pm 1.7)$. Also, the lower first molars moved significantly more forward (Lm-Gn) in U5/L5 Group 4 (4.34 \pm 3.13 ) than in the non-extraction Group 1 (0.02 \pm 2 ). (Table 6 and Figure 7).

Growth affected the amount of pre- to post treatment change in all vertical measurements and generally resulted in significant differences between growing and non-growing subjects within the same group. Example are provided in Figure 8 demonstrating that

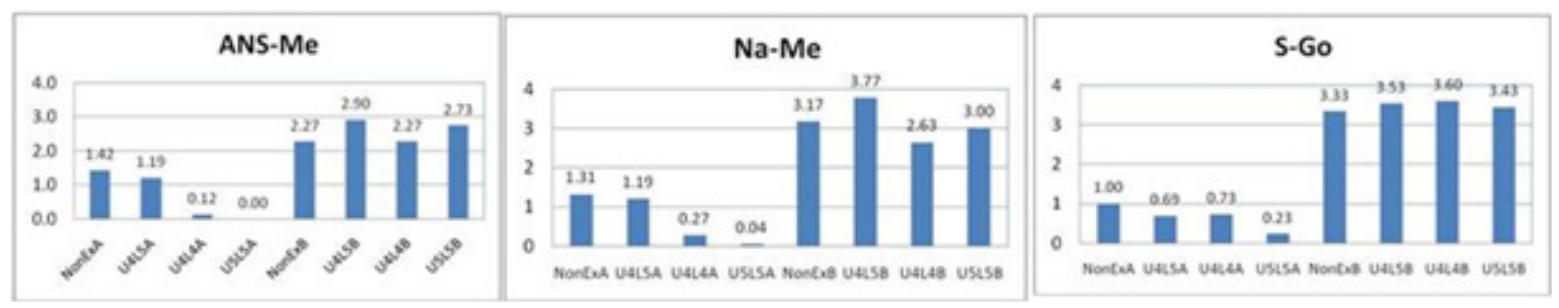

Figure 8: Bar charts demonstrating greater change during therapy for growing subjects in all 4 groups (B) compared to nongrowing subjects in all 4 groups (A) for vertical height measurements ANS-Me, Na-Me and S-Go.

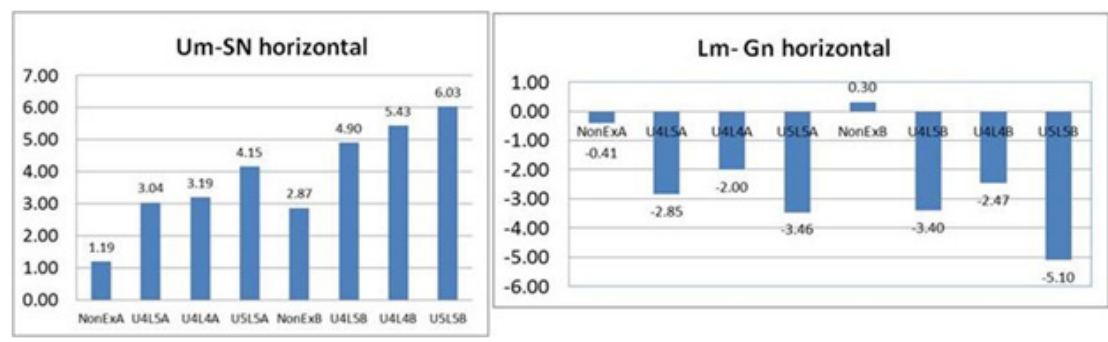

Figure 9: Bar charts demonstrating greater change during therapy for growing subjects in all 4 groups (B) compared to nongrowing subjects in all 4 groups (A) for vertical height measurements ANS-Me, Na-Me and S-Go. 
greater changes were found in growing subjects compared to non-growing subjects. (Figure 8)

Growth was also a significant factor is the analysis of horizontal molar movement change. The mean horizontal distance change of maxillary first molar (Um-SN horizontal) and mandibular first molar (Lm-Gn horizontal) was generally larger in growing compared to non-growing subjects. (Figure 9).

\section{DISCUSSION}

The purpose of the present study was to evaluate and compare the vertical changes occurred in patients treated orthodontically without or with different patterns of premolars extraction. Twelve cephalometric measurements were used that would document the vertical dimension changes during active orthodontic treatment with different patterns of extraction and non extraction.

Among four groups, comprised of 13 adult and 15 adolescent growing subjects, significant differences in first molar mesial movement were found; U5/L5 extraction Group 4 demonstrated significantly greater mesial first molar movement than in the non extraction Group 1. Angular cephalometric measurements show no significant difference among the groups tested. These findings are consistent with Al-Nimri' who found that second premolar extraction subjects showed more mesial movement of maxillary and mandibular first molars and subjects undergoing extraction of first or second premolar showed there was no significant change in MM angle.

Vertical dimensions recorded with linear measurements did not show significant different among the four groups tested either because of growth or different mechanics (intermaxillary elastics) used in closing the space. These results were consistent with Staggers, ${ }^{6}$ Sharm, ${ }^{10}$ Al-Nimri, $\mathrm{Kim}^{13}$ and Kocadereli ${ }^{8}$ who showed that there was no significant difference in the vertical dimension changes between first premolar extraction and non-extraction groups.
Growing and non growing subjects per group were compared in the present study. Vertical dimensions as measured with linear measurements increased in both growing and non growing subjects. It may be surmised that increased vertical dimensions in the non-growing adult was due mostly to the orthodontic mechanics employed and the use of intermaxillary elastics to close the space and to correct the molar relation to an Angle Class I molar relationship.

Angular measurements between growing and non growing subjects per group were not significantly different after active treatment either with extraction or without extraction. This finding is interpreted to mean that growth did not affect significant change in the vertical dimension as assessed by angular measurement. The results of the present study do not support the hypothesis that mandibular premolar extraction is associated with significant mandibular overclosure or reduction in the vertical dimension.

\section{CONCLUSION}

- Orthodontic treatment either with a non-extraction approach or with different patterns of premolar extraction led to a statistically significantly increase in the anterior facial height.

- Second premolar extraction was associated with more forward movement of the first molars.

- There was no significant change in maxillary to mandibular (MM) angle with or without extraction therapy.

Based upon the conditions of the present study, growth does not contribute to significant change in cephalometric angular measurements assessing vertical dimension of orthodontically treated patients. 


\section{REFERENCES}

1. Brandt S., Safirstein GR. (1975). Different extractions for different malocclusions. American Journal of Orthodontics and Dentofacial Orthopedics. 68, 15-41.

2. Schudy F.F. (1964). Vertical growth versus anteroposterior growth as related to function and treatment. Angle Orthodontist. 34,75-93.

3. Schudy F.F. (1965) .The rotation of the mandible resulting from growth: its implication in orthdontic treatment. Angle Orthodontist. 35 ,36-50.

4. Schudy F.F. (1968). The control of vertical overbite in clinical orthodontics. Angle Orthodontist. 38, 19-39.

5. Sassouni V., Nanda S. (1964) Analysis of dentofacial vertical proportions. American Journal of Orthodontics and Dentofacial Orthopedics. $50,801-823$.

6. Staggers JA. (1994). Vertical changes following first premolar extractions. American Journal of Orthodontics and Dentofacial Orthopedics. $105,19-24$.

7. Chua A.L, Lim J.Y.S, Lubit E.C (1993). The effect of extraction versus non-extraction orthodontic treatment on the growth of the lower anterior facial height. American Journal of Orthodontics and Dentofacial Orthopedics. 104, $361-368$.

8. Kocadereli I. (1999). The effect of first premolar extraction on vertical dimension. American Journal of Orthodontics and Dentofacial Orthopedics. 116, 41-45.

9. Al-Nimri KS. (2006). Vertical changes in Class II division 1 malocclusion after premolar extraction. Angle Orthodontist. 76, 52-58.

10. Sharma A. , Hariyani H., Darshan V. (2014). Effect of extraction versus non-extraction orthodontic treatment on nterior facial height in Class II division 1 malocclusion. National Journal of Integrated Research and Medicine. 5(1), 100-105.

11. Baccetti T. (2005). The cervical vertebral maturation (CVM) method for the assessment of optimal treatment timing in dentofacial orthopedics. Seminars in Orthodontics. 11, 119-129.

12. Aras A. (2002). Vertical changes following orthodontic extraction treatment in skeletal open bite subjects. European Journal Orthodontics. 24, 407-416.

13. Kim T-K. (2005). First or second premolar extraction effects on facial vertical dimension. Angle Orthodontist. 75, $177-182$. 